\title{
Pion Physics at Finite Volume
}

\author{
Jie $\mathrm{Hu}^{*}$ \\ Department of Physics, Duke University, Box 90305, Durham, NC 27707, USA \\ E-mail: hujie@phy.duke.edu
}

\section{Fu-Jiun Jiang}

Institute for Theoretical Physics, Bern University, Sidlerstrasse 5, CH-3012 Bern, Switzerland E-mail: fjjiangeitp.unibe.ch

\section{Brian C. Tiburzi}

Maryland Center for Fundamental Physics, Department of Physics, University of Maryland,

College Park, Maryland 20742-4111, USA

E-mail: betiburz@umd.edu

\begin{abstract}
We use chiral perturbation theory coupled to electromagnetism in a periodic box to study the impact of volume corrections on pion physics in lattice QCD. We demonstrate that conserved currents can be additively renormalized by infrared effects and construct gauge invariant single particle effective theories to explain these results. In such theories, current renormalization arises from operators involving the zero mode of the gauge field. No contradictions with Ward identities, or low-energy theorems are encountered. We also investigate finite volume corrections to the Compton scattering tensor to study the extraction of pion electromagnetic polarizabilities from lattice QCD. We argue, however, that such results cannot be used to ascertain volume corrections to polarizabilities determined in lattice QCD with background field methods. Connection is lacking because momentum expansions are not permitted in finite volume. Our argument also applies to form factors.
\end{abstract}

The XXVI International Symposium on Lattice Field Theory

July 14 - 19, 2008

Williamsburg, Virginia, USA

\footnotetext{
${ }^{*}$ Speaker.
} 


\section{Introduction}

Today lattice gauge theory [1] is a mature field. Lattice QCD can be employed to determine QCD observables from first principles using non-perturbative numerical techniques. The finite spacetime volume of the lattice is one source of systematic errors in these numerical simulations. To address the systematic errors the study of field theories in finite volume is of practical utility. We focuse our research on pions.

Recent work [2] suggests that electromagnetically gauge invariant amplitudes at finite volume may differ from their infinite volume form. Specifically investigated was the finite-size scaling of nucleon electromagnetic and spin polarizabilities that arise in nucleon Compton scattering (see, e.g., $[3,4])$. In infinite volume, the zero-frequency Compton amplitude is fixed by gauge invariance to be proportional to the total charge squared. However in finite volume, we find modificaitons for pion Compton scattering [5] at zero frequencies which suggest a finite volume renormalization of the basic interaction between the photon and the hadron's charge.

Electromagnetic polarizabilities encode fundamental properties of bound states. Pion polarizabilities can be calculated from chiral peturbation theory ( $\chi$ PT) [6] in terms of a few low-energy constants [7]. However, the predictions from $\chi$ PT do not agree with experimental results. It is unclear whether the disagreement has its roots in the experimental analysis, or in the behavior of the chiral expansion. As a first principles method, lattice QCD can be employed to determine pion polarizabilities. To this end, we perform a one-loop analysis of the quenching and partial quenching effects, as well as the volume dependence of pion Compton scattering [8]. As polarizabilities are the coefficients at second order in an expansion in photon momentum $\omega$, one would naively expect that finite volume corrections to polarizabilities can be determined from momentum expanding the finite volume Compton tensor. We find this is not the case.

\section{2. $\chi$ PT, Q $\chi$ PT and PQ $\chi$ PT for Pions}

Chiral perturbation theory provides an effective theory of low-energy QCD and is written in terms of a coset field $\Sigma=\exp (2 i \Phi / f)$ which parametrizes the Goldstone manifold. At leading order in $p^{2}$ the Lagrangian is

$$
\mathscr{L}=\frac{f^{2}}{8} \operatorname{tr}\left(D_{\mu} \Sigma^{\dagger} D^{\mu} \Sigma\right)+\lambda \frac{f^{2}}{4} \operatorname{tr}\left(m_{Q}^{\dagger} \Sigma+\Sigma^{\dagger} m_{Q}\right)
$$

The $U(1)$ gauge field, $A_{\mu}$, appears in the action of the covariant derivative, $D_{\mu} \Sigma=\partial_{\mu} \Sigma+i e A_{\mu}[Q, \Sigma]$. We work in the isospin limit, $m_{u}=m_{d}$, for simplicity.

In current lattice calculations, valence and sea quarks are often treated differently. In the rather extreme approximation known as quenched QCD (QQCD), the sea quarks are completely absent. Less extreme is partially quenched QCD (PQQCD), where sea quarks are retained but have different masses than their valence counterparts. While both approximations are certainly contrary to nature, the latter retains QCD as a limit. PQ $\chi \mathrm{PT}$ and $\mathrm{Q} \chi \mathrm{PT}$ describes the low-energy dynamics of PQQCD and QQCD, respectively. Our calculations address both quenching and partial quenching theories. 


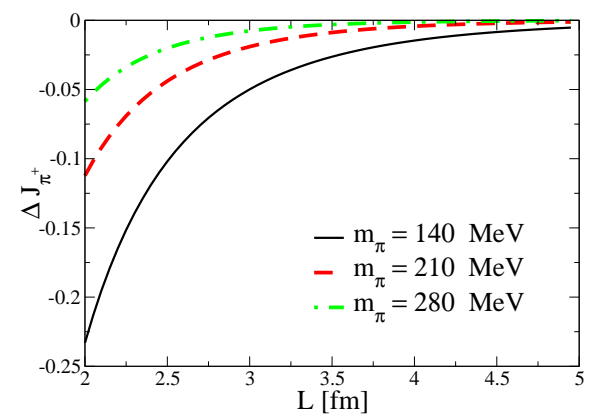

Figure 1: Finite volume screening of the charged pion current.

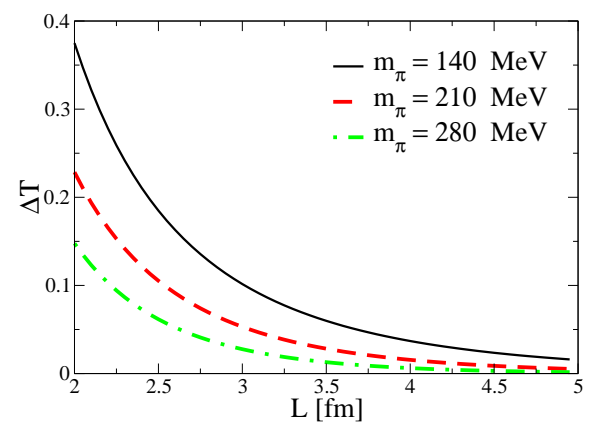

Figure 2: Finite volume zero frequency neutral pion Compton amplitude.

\section{Current Renomalization in Finite Volume}

In the limit of zero frequency and infinite volume, the current matrix element between charged pion states is required by gauge and Lorentz invariance to be

$$
\left\langle\pi^{ \pm}(P)\left|J_{\mu}\right| \pi^{ \pm}(P)\right\rangle= \pm 2 e P_{\mu},
$$

where $2 m_{\pi}$ in the right hand side is a renormalization factor. In finite volume, because of $S O(4)$ breaking, the spacial component of current is not simply the charge times the velocity. Instead, the current is screened by finite volume effects, $J=( \pm e)[1+\Delta J(L)] P$. In Fig. 1, we plot this finite volume modification [5] as a function of the box size $L$.

In the limit of zero frequency and infinite volume, the Compton tensor is sensitive only to the longest ranged electromagnetic interaction

$$
T_{\mu \nu}=2(Q e)^{2} g_{\mu \nu},
$$

For neutral pion, the Compton tensor is naively expected to be zero, but in finite volume it has nonzero contributions. In Fig. 2 we plot the neutral pion finite volume amplitude. In both figures the finit volume effect is exponentially suppressed in asymptotic volume.

These finite volume results are not anticipated from infinite volume gauge invariance. In our paper [5], however, we construct gauge invariant single particle effective theories which couple to zero mode of the gauge field and demonstrate that such finite volume results are completely consistant with Ward-Takahashi identities valid in finite volume. 


\section{Pion Polarizabilities and Volume Effects in Lattice QCD}

In infinite volume Compton scattering amplitude for a real photon to scatter off a pion can be parametrized as

$$
\begin{aligned}
T_{\gamma \pi}= & 2 m_{\pi}\left[\left(-\frac{e^{2} Q_{\pi}^{2}}{m_{\pi}}+4 \pi \alpha_{E} \omega^{2}\right) \varepsilon^{\prime *} \cdot \varepsilon+4 \pi \beta_{M} \omega^{2}\left(\varepsilon^{\prime *} \times \hat{k}^{\prime}\right) \cdot(\varepsilon \times \hat{k})\right] \\
& +\frac{e^{2} Q_{\pi}^{2}}{2 m_{\pi}^{2}} \omega^{2}\left(\varepsilon^{\prime *} \cdot \hat{k}\right)\left(\varepsilon \cdot \hat{k}^{\prime}\right)(1-\cos \theta)+\ldots,
\end{aligned}
$$

where in the center-of-momentum frame the photon momenta are $k_{\mu}=(\omega, \omega \hat{k})$ for the initial state, $k_{\mu}^{\prime}=\left(\omega, \omega \hat{k}^{\prime}\right)$ for the final state and $\cos \theta=\hat{k}^{\prime} \cdot \hat{k}$. The electric and magnetic polarizabilities are the first structure dependent terms in the low-energy expansion of the Compton scattering amplitude. We determine $\alpha$ and $\beta$ at one loop order in $\chi \mathrm{PT}, \mathrm{PQ} \chi \mathrm{PT}$ and $\mathrm{Q} \chi \mathrm{PT}$ at infinite volume [8].

We deduce the Compton scattering amplitude in a finite box for both charged and neutral pions for the process $\gamma \pi \rightarrow \gamma \pi$ to one loop order in our paper [8] for both partially quenched QCD and quenched QCD simulations. In finite volume, however, the decomposition of the Compton tensor in Eq. (4.1) is no longer valid. $S O(4)$ breaking makes the results frame dependent. Moreover, we find there are many terms in the finite volume Compton tensor not anticipated by infinite volume gauge invariance. All terms are form factors in $\omega L$, where $L$ is the spatial size of the lattice. Because of momentum quantization $(\omega=2 \pi n / L)$, these form factors cannot be expanded in $\omega L$. Thus the connection between the frequency expansion and the polarizabilities is lost. As polarizabilities are typically calculated in lattice QCD using background field methods [2,9-11], this means we cannot use the finite volume Compton tensor to deduce finite volume corrections to polarizabilities extracted from background field correlation functions. The same problem exists for electromagnetic moments. Their volume effects cannot be deduced from series expanding finite volume electromagnetic form factors about zero momentum transfer.

\section{Acknowledgments}

This work is supported in part by the U.S. Dept. of Energy, Grant Nos. DE-FG02-05ER-413680 (J.H. and B.C.T.), DE-FG02-93ER-40762 (B.C.T), and by the Schweizerischer Nationalfonds (F.-J.J.).

\section{References}

[1] T. DeGrand and C. DeTar, Lattice Methods for Quantum Chromodymamics (World Scientific, 2006)

[2] W. Detmold, B. C. Tiburzi, and A. Walker-Loud, Phys. Rev. D73, 114505 (2006), hep-lat/0603026

[3] C. E. hyde-Wright and K. de jager, Ann. Rev. Nucl. Part. Sci. 54, 217 (2004), nucl-ex/0507001

[4] M. Schumacher, Prog. Part. Nucl. Phys. 55, 567 (2005), hep-ph/0501167

[5] J. Hu, F.-J. Jiang and B. C. Tiburzi, Phys. Lett. B653, 350(2007), arXiv:0706.3408[hep-lat]

[6] J. Gasser and H. Leutwyler, Ann. Phys. 158, 142 (1984)

[7] B. R. Holstein, Comments Nucl. Part. Phys. A19, 221 (1990) 
[8] J. Hu, F.-J. Jiang and B. C. Tiburzi, to appear in Phys. Rev. D

[9] F. Fucito, G. Parisi, and S. Petrarca, Phys. Lett. B115, 148 (1982)

[10] G. Martinelli, G. Parisi, R. Petronzio, and F. Rapuano, Phys. Lett. B116, 434 (1982)

[11] C. W. Bernard, T. Draper, K. Olynyk, and M. Rushton, Phys. Rev. lett. 49, 1076 (1982) 